\title{
Erratum to: Mean Square Geodesic Deviation in the Zeldovich Problem on Light Propagation in a Universe with Inhomogeneities
}

\author{
D. D. Sokoloff ${ }^{a, b}$, A. A. Chikina ${ }^{a}$, and E. A. Illarionov ${ }^{a, b}$, * \\ ${ }^{a}$ Moscow State University, Moscow, Russia \\ ${ }^{b}$ Moscow Center for Fundamental and Applied Mathematics, Moscow, Russia \\ *e-mail: egor.mypost@gmail.com \\ Received June 21, 2021; revised June 21, 2021; accepted June 21, 2021
}

DOI: $10.1134 / \mathrm{S} 1063772921340035$

The article "Mean Square Geodesic Deviation in the Zeldovich Problem on Light Propagation in a Universe with Inhomogeneities", written by D.D. Sokoloff, A.A. Chikina, and E.A. Illarionov, was originally published electronically in Springer-Link on 17 May 2021 without Open Access. After publication in volume 65, issue 5, pages 362-369 the authors decided to make the article an Open Access publication. Therefore, the copyright of the article has been changed to (C) The Author(s), 2021 and the article is forthwith distributed under the terms of a Creative Commons Attri- bution 4.0 International License (http://creativecommons.org/licenses/by/4.0/, CC BY), which permits use, duplication, adaptation, distribution and reproduction of a work in any medium or format, as long as you cite the original author(s) and publication source, provide a link to the Creative Commons license, and indicate if changes were made.

The original article can be found online at https://doi.org/10.1134/S1063772921050073 